\title{
Tacrolimus nanomedicince in the treatment of atopic dermatitis
}

\begin{abstract}
Atopic dermatitis (AD) is a chronic, relapsing inflammation of the skin that usually affects children as well as adults. It is characterized by erythema, pruritus, exudation, scaling, crusting, and sometimes lichenification. The etiology of atopic dermatitis is unknown. Most individuals who are affected have a personal or family history of allergies. The first-line for treatment for atopic dermatitis is usually topical corticosteroids \& oral antihistamines. Nonetheless, they can be associated with tremendous adverse effects when used for longer periods of time. Tacrolimus, an immunomodulator originally used to prevent organ transplant rejection, has been formulated topically in a lipid nanoparticle and has been shown to decrease pruritis and inflammation, significantly, within three days of starting therapy and to have persistent effects for as long as 12 months. The drug was isolated from the fermentation broth of Streptomyces tsuku-baensis and belongs to a class of drugs called topical calcineurin inhibitors (TCIs).
\end{abstract}

Volume 4 Issue I - 2017

\author{
Rowany T Kakwere,' Charles Poku-Mensah, ${ }^{2}$ \\ Leigh J Mack, ${ }^{3}$ Lisa Adams, ${ }^{4}$ Vinicius Tieppo \\ Francio, ${ }^{5}$ Tony L Brown ${ }^{6}$ \\ 1,2 Major General Hugh G. Robinson Center for \\ Neuropsychiatric Studies, USA \\ ${ }^{3}$ American Biotech Labs, USA \\ 4,5 University of Science, Arts and Technology, USA \\ ${ }^{6}$ Columbia College of Physicians and Surgeons, USA
}

Correspondence: Tony L Brown, Columbia College of Physicians and Surgeons, USA, Email tbrown@post.harvard.edu

Received: April 13, 2016 | Published: January 26, 2017

Keywords: nanoparticles, nanomedicine, nanotechnology, Nanodermatology, atopic dermatitis;, tacrolimus, corticosteroids, drug delivery

Abbreviations: TCIs, topical calcineurin inhibitors; AD, atopic dermatitis; nm, nanometer(s); FK506, tacrolimus; IL 8, interleukin 8; FKBP 12, protein receptor macrophilin-12; NLC, nanostructured lipid carrier

\section{Objective}

To assess the efficacy and safety of tacrolimus for use in the treatment of atopic dermatitis.

\section{Areas covered}

This review covers the pathophysiology of atopic dermatitis, available medications and its associated challenges in treatment. Newer approaches in the treatment of atopic dermatitis treatment such as the use of nanomedicines, and in this case, topically formulated tacrolimus.

\section{Introduction}

\section{Atopic dermatitis}

Atopic dermatitis (AD) is a chronic, relapsing inflammation of the skin that usually affects children as well as adults. Atopic dermatitis is mostly referred to as 'eczema', which is a common term for the different of types of inflammation of the skin. Atopic dermatitis is essentially the most common of the many varieties of eczema. Symptoms may vary in presentation and severity from individual to individual. An individual affected with this skin condition usually experiences redness of the skin, intense itching, exudation and dryness of the skin. Sometimes, hardening and leathering of the skin occurs due to chronic irritation of the skin. Typically, there are durations of time when the disease is worse, referred to as flares or exacerbations, followed by means of intervals when the epidermis improves or clears up thoroughly, known as remissions. As some children affected with this skin condition mature, their skin disease disappears or improves, despite the fact that their dermis more often than not remains easily irritated and dry. In others, this skin condition remains to be a gigantic challenge in adulthood. The etiology of atopic dermatitis is unknown. Most individuals who are affected have a personal or family history of allergies. It is assumed that genetics, environmental factors and hereditary factors play a role in the manifestation of this skin condition. The main objectives in the treatment of atopic dermatitis are to rehydrate the skin and keep it moisturized, prevent flares and heal the skin. Topical corticosteroids and oral antihistamines are the mainstay of treatment for atopic dermatitis. Nonetheless, they can be associated with tremendous adverse effects when used for longer periods of time. Some of the adverse effects encountered are skin thinning (atrophy), striae (stretch marks), skin discoloration, telangiectasia (enlarged blood vessels), easy bruising and tearing of the skin, infections and hypertrichosis.

\section{Nanomedicine}

Nanotechnology, more commonly, is the use and application of materials with sizes in the nanometer variety. It is quickly being made use of in medicine. Nanomedicine is simply the use of nanotechnologies in a healthcare environment and the vast majority of benefits which have already been noticeable involve the usage of nanoparticles to improve the way drug substances behave. The nanomedicines that are currently on hand are overcoming a few of the down sides faced by using natural clinical methods in delivering the benefit from the drug molecules used. Nanomedicines, for that reason, play a principal function in ensuring enough of the drug enters the body, that drug that does enter stays in the body for longer periods and is targeted mainly to the areas that require treatment. Nanodermatology focuses on the use of nanomaterials (sized less than 100nm) on the skin. Nanotechnology is being used in cutaneous drug delivery to stabilize compounds, allow controlled release, and to enable targeted drug localization to maximize activity and minimize toxicity.

\section{Tacrolimus}

One technique of nanotechnology in medicine involves employing nanoparticles to deliver medicinal drugs. Particles are engineered in order to be attracted to infected cells, which make it possible for direct 
healing of these cells. This system reduces injury to healthy cells within the body and enables for earlier detection of disease. Tacrolimus, an immunomodulator originally used to prevent organ transplant rejection, has been formulated topically in a lipid nanoparticle. This modified form is more soluble than the unmodified form which is unencapsulated. It offers better retention and delivery kinetics in the skin. These characteristics ensure reduced systemic release and toxicity, greater efficacy, and an extended duration of action. The unwanted effects of topical corticosteroids on hormonal function are the main concerns in long-term topical ointment therapy in patients affected by atopic dermatitis. Topically formulated tacrolimus, not only go with existing treatment plans but also get over a few of the downsides of topical ointment corticosteroid remedy and match the long-term needs of patients in protecting against disease progression.

Tacrolimus mechanism of action: Tacrolimus (FK506) is a macrolide immunosuppressant isolated from the fermentation broth of Streptomyces tsuku-baensis and is potent towards atopic dermatitis. The inflammation seen in atopic dermatitis is caused by the activation of the immune system though the reason behind the activation is unknown. Tacrolimus suppresses the immune system and the inflammation by inhibiting the enzyme (calcineurin) responsible for the multiplication of cells needed for activation of the immune system. Tacrolimus decreases peptidyl-prolyl isomerase activity by binding with high affinity to the FKBP-12, i.e, protein receptor macrophilin-12. The resulting drug-protein complex inhibits a calcium-dependent phosphatase transmitting chemical termed calcineurin, which leads to blockade of signal transduction target cells which result in blockade of the release of $\mathrm{TH} 2$ cytokines from mast cells and T-cells. Topical tacrolimus may also have direct impact on keratinocytes. It seems to decrease the number of proinflammatory cytokines, particulary IL-8 cytokine receptors on the keratinocyte, for this reason reducing inflammation. In contrast to many steroids, topical tacrolimus could also be used on all body locations for longer periods of time. Glaucoma, skin atrophy, and other local risks associated with steroids do not arise, nor do the systemic adverse results such as stunted growth and adrenal-axis/hypothalamus suppression. Based on the lengthy-time period efficacy and safety, topical tacrolimus, is therefore, of curiosity to occupational dermatologists.

Side effects of topical tacrolimus: Topical tacrolimus is steroid-free. It can also be used in both adults and children. As with every drug ever made, topically formulated tacrolimus has possible side effects. A majority of patients tolerate topical tacrolimus well. Essentially, the most common side effects experienced are a feeling of heat or a sensation of burning, soreness, itching, or stinging in the area of treated skin. This is in most cases is mild to moderate in severity and clears up within a few days of commencing treatment. Other much less common side effects include muscle aches, fever, headache, flulike signs, coughs, and in treated areas acne and folliculitis (infection of the hair follicle).

\section{Discussion}

The reason why the modified nanostructured lipid carrier (NLC) was developed was to help improve the bioavailability and solubility of tacrolimus using lipophilic solubilizers. These tacrolimus-loaded nanostructured lipid carriers produce improved drug stability, increased entrapment efficiency, higher penetration and improved skin targeting effects and localization. Thus, effective treatment of atopic dermatitis occurs in a shorter amount of time. Most of the side effects which have been reported are those described on the product's label. A burning sensation at the site of application is usually the most reported adverse outcome. However, this side effect is traditionally for a short amount of time lasting from ten minutes to two hours and usually happens for the period of the first few days of therapy and gradually declines over the course of treatment. There have been reports that such discomfort may be avoided by using first utilizing a potent topical corticosteroid for five to seven days to curb the acute inflammation. Fever and flu-like symptoms are probably the most typically stated systemic adverse events. Unique attention has been given to skin infections as a capabilities complication of topical treatment with topical tacrolimus. ${ }^{1-12}$

\section{Conclusion}

According to current data, the efficacy and safety of tacrolimus in treating atopic dermatitis has been well demonstrated in countless tremendous-scale randomized controlled trials and open-label studies with follow ups of up to 3 years. Although studies did have shown tacrolimus to have side effects topical tacrolimus has less severe side effects when compared with other drugs in the market for the same therapeutic target. Tacrolimus does not have an effect on collagen synthesis nor is it metabolized in the skin, indicating that there is no potential for percutaneous interactions that would have an effect on the metabolism of tacrolimus. Over-dosage following topical administration is unlikely. Studies indicate no evidence of systemic accumulation of tacrolimus in individuals (both children and adults) treated for prolonged intervals (up to 12 months) with tacrolimus. Tacrolimus hold a greater promise for the future in nanodermatology. It has furnished its potential in improving therapeutic benefits in atopic dermatitis due to its increased therapeutic efficacy with minimal toxicity. On the groundwork of anecdotal reports, tacrolimus also seems to be efficacious in a variety of other skin conditions. However, the long-term effects of prolonged use (beyond 3 years) are uncertain, more clinical studies on the efficacy and safety of tacrolimus in the treatment of atopic dermatitis therapy over a longer period of time are required.

\section{Acknowledgements}

None.

\section{Conflict of interest}

The author declares no conflict of interest.

\section{References}

1. https://www.niams.nih.gov/health_Info/atopic_dermatitis/

2. Kumar A. Nanomedicine in drug delivery. USA: CRC Press/Taylor \& Francis Group; 2013.

3. Nasir A. Nanodermatology: A Bright Glimpse Just Beyond the Horizon - Part I. Skin Therapy Lett. 2010;15(8):1-4.

4. Nanotechnology in Medicine. (n.d.). Retrieved from http://www. understandingnano.com/medicine.html

5. Schwartz RA, Elston DM. Pediatric Atopic Dermatitis Medication Topical corticosteroids, Systemic corticosteroids, Antimicrobials, Antipruritics, Immunomodulators. 2016.

6. http://www.britishsocietynanomedicine.org/what-is-nanomedicine.html

7. McCollum AD, Paik A, Eichenfield LF. The Safety and Efficacy of Tacrolimus Ointment in Pediatric Patients with Atopic Dermatitis. Pediatr Dermatol. 2010;27(5):425-436. 
8. Leung AC, Hon KE. Atopic Dermatitis: A Review for the Primary Care Physician. USA: Nova Science Publishers Inc; 2011.

9. Rahman M, Akhter S, Ahmad J, et al. Nanomedicine-based drug targeting for psoriasis: Potentials and emerging trends in nanoscale pharmacotherapy. Expert Opin Drug Deliv. 2015;12(4):635-652.

10. https://www.medicines.org.uk/emc/medicine/8816
11. Woo DK, James WD. Topical tacrolimus: a review of its uses in dermatology. Dermatitis. 2005;16(1):6-21.

12. Undre NA, Moloney FJ, Ahmadi S, et al. Skin and systemic pharmacokinetics of tacrolimus following topical application of tacrolimus ointment in adults with moderate to severe atopic dermatitis. Br J Dermatol. 2009;160(3):665-669. 